\title{
Time Series Analysis of Dengue Incidence in Rio de Janeiro, Brazil
}

\author{
Paula M. Luz,* Beatriz V. M. Mendes, Claudia T. Codeço, Claudio J. Struchiner, and Alison P. Galvani \\ Department of Epidemiology and Public Health, Yale University, New Haven, Connecticut; Institute of Mathematics/COPPEAD, \\ Rio de Janeiro Federal University, Rio de Janeiro, RJ, Brazil; Program for Scientific Computing, Oswaldo Cruz Foundation,
} Rio de Janeiro, RJ, Brazil

\begin{abstract}
We use the Box-Jenkins approach to fit an autoregressive integrated moving average (ARIMA) model to dengue incidence in Rio de Janeiro, Brazil, from 1997 to 2004. We find that the number of dengue cases in a month can be estimated by the number of dengue cases occurring one, two, and twelve months prior. We use our fitted model to predict dengue incidence for the year 2005 when two alternative approaches are used: 12-steps ahead versus 1-step ahead. Our calculations show that the 1-step ahead approach for predicting dengue incidence provides significantly more accurate predictions ( $P$ value $=0.002$, Wilcoxon signed-ranks test) than the 12 -steps ahead approach. We also explore the predictive power of alternative ARIMA models incorporating climate variables as external regressors. Our findings indicate that ARIMA models are useful tools for monitoring dengue incidence in Rio de Janeiro. Furthermore, these models can be applied to surveillance data for predicting trends in dengue incidence.
\end{abstract}

\section{INTRODUCTION}

Dengue fever adversely impacts the health, social, and economic status of many tropical countries of the world. ${ }^{1-3}$ In the past three decades, dengue has expanded throughout the Americas with increasing incidence. ${ }^{4,5}$ The Southeast region of Brazil and, in particular, the city of Rio de Janeiro, have been most affected by dengue. ${ }^{6,7}$ In Rio de Janeiro, dengue epidemics of increasing magnitude and severity occur every three to four years beyond the endemic levels. ${ }^{8,9}$ Although dengue occurs throughout the year, cases peak from January to $\mathrm{May}^{7,10}$ during the hot-wet season. Field studies of the city's Aedes aegypti population show that the vector peaks concurrently. ${ }^{11}$

Public health surveillance oversees the ongoing collection, analysis, and interpretation of disease data. The goal is to monitor and predict trends in infectious disease incidence to facilitate early public health responses to minimize morbidity and mortality. Accordingly, dengue surveillance seeks to detect rising trends early enough to reduce further transmission and/or to prepare for increased hospital demand. ${ }^{12}$ Dengue surveillance is usually based on a passive system, that is, the reporting of dengue cases by health professionals to the health authorities is mandatory by law. This system, however, does not perceive changes in incidence early enough for adequate response. ${ }^{12}$ Aside from monitoring trends in disease incidence; dengue surveillance can also focus on measures of mosquito abundance, which are based on the many life-stages of the mosquito. However, these latter measures are laborious and have not consistently been correlated with dengue incidence. $^{12}$

It has been proposed that climate variables can increase the predictive power of dengue models. ${ }^{13}$ The relationship between climate and dengue has been assessed in multiple settings using different statistical methods. ${ }^{14-25}$ Increased temperature has been associated with dengue in Thailand, ${ }^{21,23}$ Indonesia, ${ }^{14,19,25}$ Singapore, ${ }^{16}$ Mexico, ${ }^{18}$ and Puerto Rico. ${ }^{22}$ Rainfall has been found to correlate with dengue in Indonesia, ${ }^{19}$ Trinidad, ${ }^{17}$ Venezuela, ${ }^{15}$ Barbados, ${ }^{20}$ and Thailand. ${ }^{23}$ Furthermore, mosquito population dynamics vary for differ-

* Address correspondence to Paula M. Luz, Department of Epidemiology and Public Health, Yale University, 60 College Street, New Haven, CT 06511. E-mail: paula.luz@yale.edu ent geographic regions where dengue is transmitted suggesting that the influence of climate on dengue may be sitespecific. $^{26}$ Statistical tools used to measure the association of climate and dengue in these studies have included graphic assessments, ${ }^{19,25}$ correlation coefficients, ${ }^{14,15,17,20,21}$ linear univariate regression, ${ }^{16,20}$ linear multivariate regression, ${ }^{14,18,22,23}$ and time series analysis. ${ }^{24}$

Autoregressive integrated moving average (ARIMA) models, which use time series analyses, are particularly useful in modeling the temporal dependence structure of a time series as they explicitly assume temporal dependence between observations. ${ }^{27}$ Through the modeling of the temporal structure, particularly for seasonal diseases, predictions made with ARIMA models have been shown to be more accurate than those obtained by other statistical methods. ${ }^{28-31}$ Predictions can be made for a certain number of steps ahead, with this number being given by the highest order of the parameters of the model. ${ }^{27}$ The ARIMA models have been used successfully in epidemiology to monitor and predict infectious diseases, such as malaria and hepatitis A incidence, ${ }^{31}$ influenza and pneumonia deaths, ${ }^{28}$ as well as other infectious diseases incidences, ${ }^{32-36}$ and the use of health facilities, ${ }^{37}$ or isolation beds. 38

In this study, we used ARIMA models to monitor and predict dengue incidence in Rio de Janeiro. Specifically, we used the Box-Jenkins approach ${ }^{39}$ to fit an ARIMA model to dengue incidence from 1997 to 2004. The fitted model was then used to predict dengue incidence for the year 2005. We used two approaches to estimate the year 2005 predicted values: 12-steps ahead and 1-step ahead. The predictive power of the two approaches was assessed by calculating the root mean squared error (RMSE), ${ }^{13}$ and the statistical difference of the errors of the two approaches was tested by Wilcoxon signedranks test. ${ }^{40}$ Furthermore, we evaluated whether alternative models incorporating climate variables as external regressors have greater predictive power. We estimated the correlation coefficients between dengue incidence and climate variables, and incorporated climate variables that significantly correlate with dengue incidence in alternative ARIMA models. Our ARIMA model closely fits dengue incidence in Rio de Janeiro. The 1-step ahead approach for predicting dengue incidence provides significantly more accurate predictions $(P=$ $0.002)$ than the 12 -steps ahead approach. In addition, ARIMA models in which lag-0 maximum temperature and 
lag-1 number of rainy days were independently incorporated as external regressors have improved predictive power, although the improvement is not statistically significant.

\section{MATERIALS AND METHODS}

Rio de Janeiro occupies an area of $1182 \mathrm{~km}^{2}$, is located at $22^{\circ} 54^{\prime} \mathrm{S}$ and $43^{\circ} 14^{\prime} \mathrm{W}$, and has a population of over 6 million people. The year-round climate, which is classified as tropical humid, can be divided into two seasons: the hot-wet season, from approximately December to May, and the cool-dry season, from approximately June to November. In the hot-wet season, the maximum and minimum temperatures range between 30 and $21^{\circ} \mathrm{C}$, respectively, and accumulated rainfall per month averages $145 \mathrm{~mm}$ (10-year averages). In the cool-dry season, the maximum and minimum temperatures range between 26 and $17^{\circ} \mathrm{C}$, respectively, and accumulated rainfall per month averages $117 \mathrm{~mm}$ (10-year averages). Several meteorologic stations are located within the city where temperature and rainfall are recorded daily. For our analyses, we gathered available monthly data on maximum and minimum temperatures (in degrees Celsius), accumulated rainfall (in millimeters), and number of rainy days for the years $1997-2005 .{ }^{41} \mathrm{We}$ calculated an average time series for each climate variable to represent the city-level climate and used this aggregate measure in our analyses. In addition to the climate data, we gathered monthly dengue incidence data, that is, the number of dengue cases reported to the Department of Health of Rio de Janeiro per month for the period 1997-2005. ${ }^{42}$ Dengue reporting is compulsory in Brazil, and reported dengue cases include those fulfilling a clinical classification or confirmed by laboratorial tests.

Using the dengue incidence data from 1997 to 2004, we fit an ARIMA model to dengue incidence, and then used the fitted model to out-of-sample predict dengue incidence for the year 2005. We used the Box-Jenkins approach to ARIMA modeling of time series, which consists of a four-step process. $^{27,31,32,34,39,40,43,44}$ First, we evaluated the need for variance-stabilizing transformations using the mean-range plot. Second, we determined the order of non-seasonal $(p, d, q)$ and seasonal $(\mathrm{P}, \mathrm{D}, \mathrm{Q})_{12}$ autoregressive $(\mathrm{AR})$ parameters $(\mathrm{p}$ and $\mathrm{P})$ and moving average (MA) parameters ( $\mathrm{q}$ and $\mathrm{Q})$, and the need for non-seasonal and seasonal differencing (d and D), using the following five tools: 1) The plot of dengue incidence, which assists in the need for non-seasonal and seasonal differencing; 2) The autocorrelation (ACF) and partial autocorrelation (PACF) functions, which indicate the temporal dependence structure in the stationary time series; 3) The Akaike Information Criterion (AIC), which assists in the goodness-of-fit of the model, whereas penalizing for the number of parameters; 4) The Ljung-Box test, which measures the ACF of the residuals; and, 5) the significance of the parameters, which should be statistically different from zero (that is, the $t$ statistic should exceed 2 in absolute value). Third, we estimated the parameters of the ARIMA model by maximum likelihood. Finally, we graphically compared the model's fitted values with the observed data to check if it indeed models dengue incidence.

We used the identified ARIMA model to calculate oneyear, out-of-sample predicted values and $95 \%$ prediction intervals, employing two approaches to estimate the year 2005 predicted values. The first, straightforward approach uses the fitted ARIMA model to predict the subsequent 12-steps ahead, that is, the 12 out-of-sample observations that constitute the year 2005. This approach could be adopted for dengue surveillance. However, to our view, this approach does not reflect the on-going nature of surveillance. Health departments in charge of disease monitoring continuously work with new information as it arrives. Thus, the newly collected data could easily be incorporated possibly leading to increased predictive power. Accordingly, our second approach was iterative. It entails using the ARIMA model to predict 1-step ahead, that is, the next month predicted value, January 2005. Then, as the observed value for January 2005 was obtained, we updated the data to January 2005, re-estimated the parameters of the ARIMA model, and computed the next 1 -step ahead predicted value, February 2005. This process was continued until the end of the year 2005.

We assessed the out-of-sample predictive power of the two approaches by calculating the RMSE, which measures the amount by which the fitted values differ from the observed values. ${ }^{13}$ A lower RMSE indicates increased predictive power. Moreover, we also applied the Wilcoxon signed-ranks test to statistically assess the difference in the errors between the two approaches. ${ }^{31,40}$ This test evaluates if the median of the distribution of the difference of the errors (that is, fitted minus observed value) of the two approaches is statistically different from zero at the $5 \%$ significance level.

We further evaluated whether alternative ARIMA models incorporating climate variables as external regressors have greater predictive power. ${ }^{13}$ To facilitate selection of climate variables to be used as external regressors, we computed Pearson's correlation coefficient between dengue incidence and climate variables after "pre-whitening" of the time series. $^{27,33}$ That is, we first removed the trend and seasonal components of the time series using ARIMA modeling, and the iterative steps described above. After fitting the best ARIMA model to each time series individually, we obtained the residuals of each time series, i.e., the difference between an observation and its fitted value according to the model. We then computed the correlation coefficients between the residuals of dengue incidence and the residuals of each climate time series over a range of lags. The climate variables found to correlate with dengue cases under this analysis were tested as additional regressors in alternative ARIMA models. We fit ARIMA models with external regressors to dengue incidence from 1997 to 2004, and then used the fitted models to out-ofsample predict the year 2005 using the two approaches. To measure the predictive power of models, the RMSE was calculated. To test the significance of the difference in the errors of the predictions, the Wilcoxon signed-ranks test was used.

The statistical software $\mathrm{R}$ was used for all statistical analyses (version 2.5.0 $0^{45}$ ), and graphic displays and automatic algorithms were used to aid in the selection of the ARIMA models. ${ }^{40,43,46,47}$

\section{RESULTS}

We found it necessary to stabilize the variance of dengue incidence by computing its natural logarithm. ${ }^{34}$ The transformed dengue incidence shows far less dispersion (Figure 1). All further statistical procedures, descriptive or analytic, were 


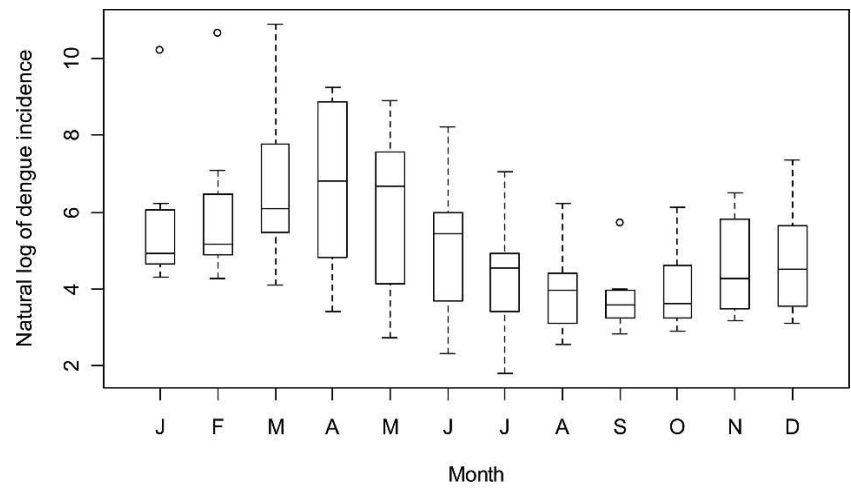

Figure 1. Natural logarithm of dengue incidence in the city of Rio de Janeiro, Brazil, for the period 1997-2004.

performed on the logarithmically transformed dengue incidence. The plot of dengue incidence shows no significant trend (Figure 2). The plots of the sample ACF and PACF describe the temporal dependence structure in dengue incidence and suggest that non-seasonal and seasonal parameters are needed in the model (Figure 2). The slow decay in the ACF at lags 1-3, associated with a PACF cutoff at lag 2 suggests a non-seasonal AR $(P=2)$. Also, the slow decay in the ACF at lags 12 and 24, associated with a PACF cutoff at lag 12, suggests a seasonal $\operatorname{AR}(P=1)$. Indeed, the best model was the multiplicative ARIMA $(2,0,0) \times(1,0,0)_{12}$ $(\mathrm{AIC}=211.97$, Table 1$)$ on the non-differenced dengue incidence (or, more precisely, the multiplicative ARMA[2,0] $\times$ $[1,0]_{12}$, because no differencing is needed). The plots of the ACF and PACF of the residuals show no remaining temporal correlation (Figure 3). We calculated the Ljung-Box test statistic and did not reject the null hypothesis of independence in the residuals time series $(P$ value $=0.373)$. The model's fitted (1997-2004) and predicted values (year 2005) follow the observed dengue incidence (Figure 4). Graphically, predicted values estimated using the 1-step ahead approach show less
TABLE 1

Coefficient, standard error, and $t$ statistic of the parameters of the ARIMA $(2,0,0) \times(1,0,0)_{12}$ model*

\begin{tabular}{lrcr}
\hline \multicolumn{1}{c}{ Parameters } & Coefficient & Standard error & $t$ statistic \\
\hline Intercept & 5.0132 & 0.6287 & 7.9242 \\
Non-seasonal AR (1) & 1.2956 & 0.0930 & 13.9382 \\
Non-seasonal AR (2) & -0.4592 & 0.0936 & -4.9040 \\
Seasonal AR (1) & 0.3721 & 0.1010 & 3.6842 \\
\hline
\end{tabular}

* Parameters estimated by maximum likelihood.

ARIMA = autoregressive integrated moving average.

dispersion from observed values $(\mathrm{RMSE}=0.5176)$ when compared with the 12-step ahead approach (RMSE = 0.6789) (Figure 4). Furthermore, predictions estimated using the 1-step ahead approach are significantly better than those estimated using the 12-steps ahead approach $(P$ value $=$ 0.002 ).

We subsequently assessed whether alternative ARIMA models incorporating climate variables as external regressors have greater predictive power. As highlighted in the Methods section, we first removed trend and seasonal components of each time series through ARIMA modeling. Then, on the basis of the correlation coefficients of the residuals of the time series over a range of lags, we identified the following as potential external regressors: lag-0 maximum temperature (Pearson correlation $=0.263, P$ value $=0.011$ ); lag-0 minimum temperature $($ Pearson correlation $=0.225, P$ value $=$ 0.030); lag-1 accumulated rainfall (Pearson correlation $=$ $0.313, P$ value $=0.002)$; and lag-1 number of rainy days $($ Pearson correlation $=0.283, P$ value $=0.006)($ Figure 5) . Alternative ARIMA models incorporating, independently, lag-0 maximum temperature and lag-1 number of rainy days are statistically supported. These models have improved predictive power as measured by the RMSE (Table 2). However, the predictions calculated with either model were not statistically better than those estimated with the ARIMA model without external regressors (irrespective of the approach used to generate predictions), indicating that climate variables did

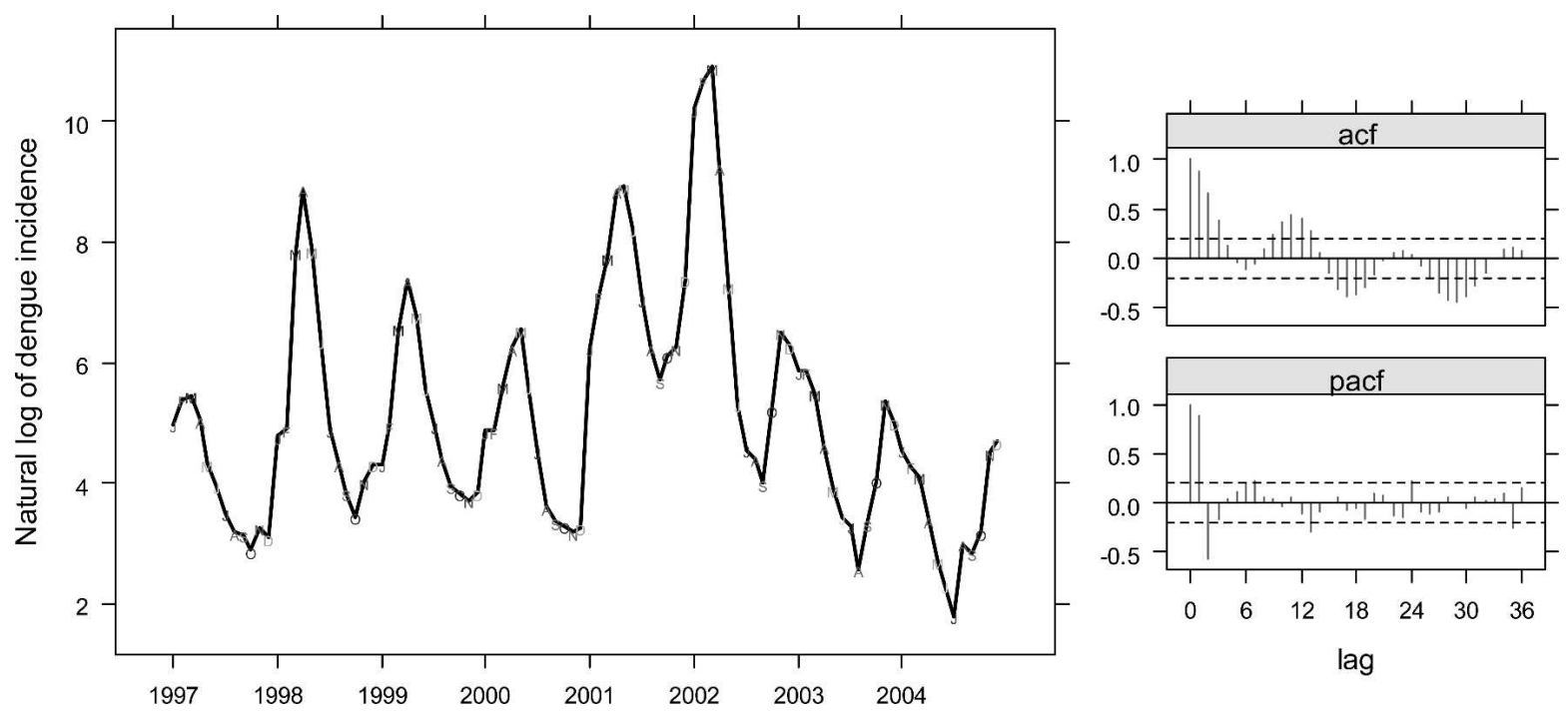

FIGURE 2. Left panel: Natural logarithm of dengue incidence in the city of Rio de Janeiro, Brazil, for the period 1997-2004. Right panels: Sample autocorrelation (acf, top) and partial autocorrelation (pacf, bottom) functions of dengue incidence; $x$-axis gives the number of lags in months. Dotted line indicates $95 \%$ confidence interval. 


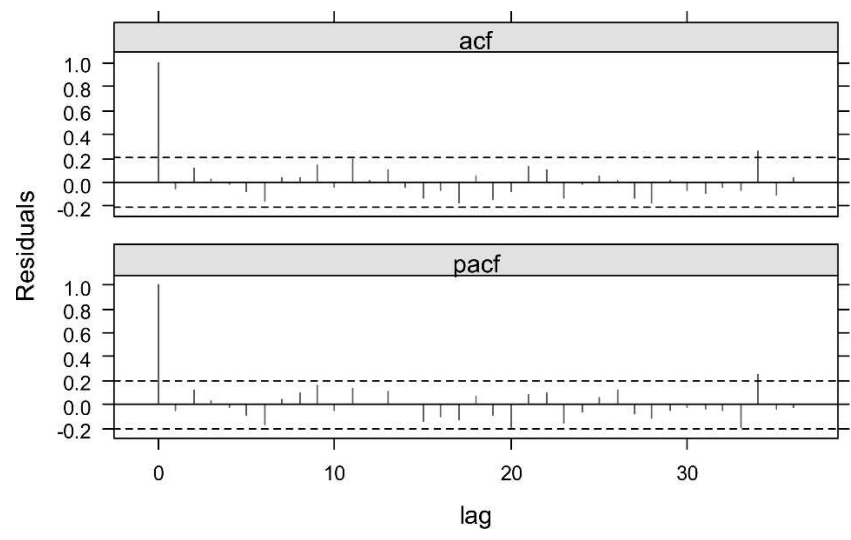

FiguRE 3. Autocorrelation (acf, top) and partial autocorrelation (pacf, bottom) of the residuals of the autoregressive integrated moving average (ARIMA) $(2,0,0) \times(1,0,0)_{12}$ model fitted to the natural logarithm of dengue incidence. The $x$-axis gives the number of lags in months. Dotted line indicates $95 \%$ confidence interval.

not provide any predictive information beyond that already given by dengue incidence.

\section{DISCUSSION}

We have reached an ARIMA model that closely fits dengue incidence in Rio de Janeiro. The autoregressor and moving average parameters of our model imply the number of dengue cases in a month can be estimated by the number of dengue cases occurring one, two, and twelve months prior. Furthermore, our model predicts dengue incidence for a subsequent year. We found that the ARIMA model's 12-steps ahead predictions agree with the observed dengue incidence. Moreover, we have explored the improvement in predictive power when applying a more realistic use of the data. Disease monitoring by public health departments entails ongoing data handling, processing, and updating. The Health Department of Rio de Janeiro is the authority to which reporting first occurs, and thus it is an appropriate level of organization for the implementation of an ARIMA predictive model, where data updating is possible. We found that model predictions are further improved in the context of the likely data availability of the Health Department. The ARIMA model's 1-step ahead predictions were significantly closer to the observed dengue incidence than the 12 -steps ahead predictions.

Temperature and rainfall affect the dengue transmission cycle in multiple ways. Temperature indirectly influences the biting frequency of $A$. aegypti both by increasing the rates of development and digestion and by decreasing the duration of the gonotrophic cycle and female size. ${ }^{48,49}$ Increased temperature accelerates viral dissemination within the mosquito, reducing the extrinsic incubation period in the vector. ${ }^{50}$ Higher temperatures increase the ratio of the standing crop of pupae to the number of adult females. ${ }^{51}$ Eggs need water to hatch, and larvae and pupae are aquatic.

Several studies have assessed the correlation between dengue and climate variables. ${ }^{18,21,24}$ In Thailand, dengue was found to correlate with lags 3-4 average temperature. ${ }^{21}$ In Taiwan, dengue was found to significantly correlate with lags 1-4 maximum temperature, lags 1-3 minimum temperature, lags 1 - relative humidity, and lag- 2 rainfall. ${ }^{24}$ For Mexico and Barbados, high correlations between dengue and climate are smeared over a range of lags. ${ }^{18,20}$

Pre-whitening facilitates the evaluation of correlation between time series. ${ }^{27,33}$ When pre-whitening is not performed, significant correlation coefficients are smeared over a range of lags because of the autocorrelation present in seasonal time series. ${ }^{27}$ Furthermore, statistics used to compute correlation coefficients, such as Pearson or Spearman correlation coefficients, assume that observations are independent and identically distributed. Thus, the use of such statistics to measure correlation between temporally dependent data will produce false results.

We computed correlation coefficients between dengue incidence and climate variables over a range of lags after prewhitening. Pre-whitening was performed by modeling each time series individually using the ARIMA model. We found that dengue incidence significantly correlated with lag-0 maximum and minimum temperatures, lag- 1 accumulated rainfall, and lag-1 number of rainy days. Conversely, if pre-whitening was not performed, dengue incidence was found to correlate with lags 0-2 maximum and minimum temperatures, although

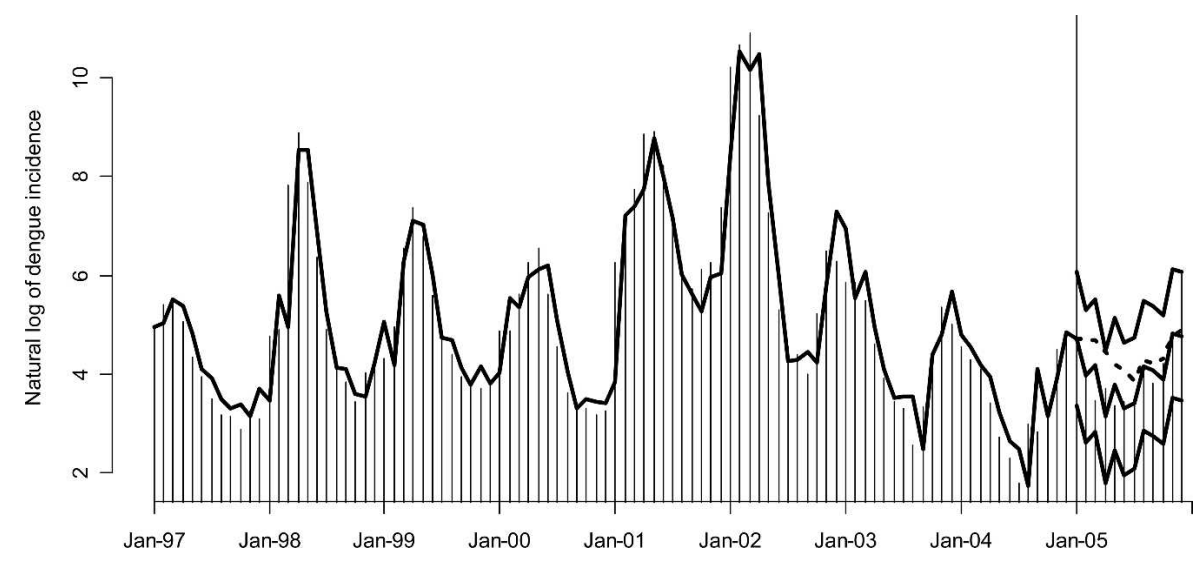

Figure 4. Columns: natural logarithm of dengue incidence in the city of Rio de Janeiro, Brazil, for the period 1997-2005. Solid lines: autoregressive integrated moving average (ARIMA) $(2,0,0) \times(1,0,0)_{12}$ model's fitted values (1997-2004) and 1-step ahead predicted values (year $2005)$ with their $95 \%$ prediction intervals. Dashed line: ARIMA $(2,0,0) \times(1,0,0)_{12}$ model's 12 -steps ahead predicted values. Vertical line separates model's fitted from predicted values. 

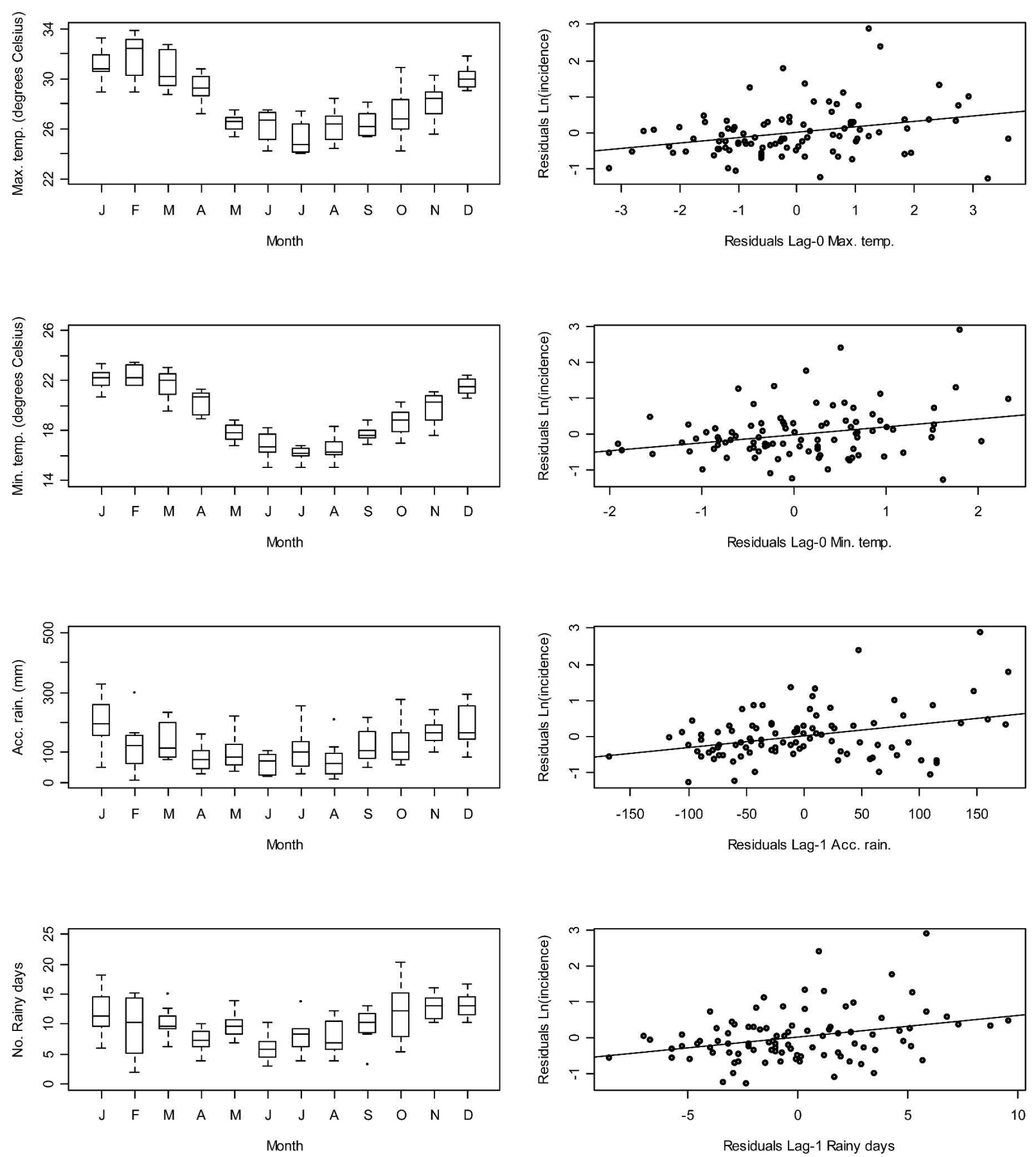

FIGURE 5. Left column: Seasonal pattern of maximum and minimum temperatures, accumulated rainfall, and number of rainy days in the city of Rio de Janeiro, Brazil, for the period of 1997-2004. Right column: Scatter plot of the residuals of dengue incidence against the residuals of lag-0 maximum temperature (1st), lag-0 minimum temperature (2nd), lag-1 accumulated rainfall (3rd), and lag-1 number of rainy days (4th).

TABLE 2

External regressors' coefficient, standard error, and $t$ statistic of three ARIMA $(2,0,0) \times(1,0,0)_{12}$ models*

\begin{tabular}{lcccc}
\hline \multicolumn{1}{c}{ ARIMA models } & Coefficient & Standard error & $t$ statistic & RMSE \\
\hline None & & & & 0.5176 \\
Lag-0 max. temperature & 0.0867 & 0.0344 & 2.5190 & 0.5107 \\
Lag-1 rainy days & 0.0242 & 0.0110 & 2.2030 & 0.4253 \\
\hline $\begin{array}{l}\text { * Last column gives the predictive power as measured by the root mean squared error } \\
\text { (RMSE) of the three autoregressive integrated moving average (ARIMA) models using the } \\
\text { 1-step ahead approach. Parameters estimated by maximum likelihood. }\end{array}$ &
\end{tabular}

no significant correlation was detected for accumulated rainfall or number of rainy days at any lag.

The identification of climate variables that significantly correlated with dengue incidence allowed us to test alternative ARIMA models incorporating these variables as external regressors. We found that ARIMA models incorporating, independently, lag-0 maximum temperature and lag-1 accumulated rainfall were statistically supported. Although these models have improved predictive power, the difference was 
not significant when compared with the ARIMA model with no external regressors. It is arguable that because these variables are readily available, the slight increased predictive power might justify their inclusion in predictive models. In Taiwan, for example, time series models incorporating climate variables as external regressors showed improved fit to data. $^{24}$

Our study is the first to assess the temporal dependence structure of dengue incidence for Rio de Janeiro. Previous studies have followed the subsequent viral introductions, ${ }^{6}$ assessed the sero-epidemiologic profile of the population, ${ }^{52,53}$ and examined the ecology of $A$. aegypti population. ${ }^{54-57}$ Furthermore, descriptive analyses of the temporal characteristics of dengue occurrence have shown both that dengue is endemic with epidemics of increasing magnitude occurring every three-to-four years, and that epidemics occur during the hot-wet season, from January to May. ${ }^{7,10}$ Thus, our study complements the understanding of dengue incidence in this setting through a characterization of the temporal dependence structure of the disease.

There is an urgent need for improved approaches for monitoring and predicting dengue incidence to reduce the substantial morbidity and mortality caused by this disease. ${ }^{58}$ Additionally, dengue incidence is increasing and spreading geographically, ${ }^{6,59}$ such as in Brazil. Nonetheless, adequate dengue surveillance methods are still lacking. Dengue surveillance through the monitoring of the vector has been shown to correlate poorly with dengue incidence. ${ }^{7,60}$ Our modeling approach can be used to monitor and predict dengue incidence in Rio de Janeiro. The ARIMA model could be used to optimize dengue prevention by providing estimates on dengue incidence trends. Accurate predictions for even a few months ahead provide an invaluable opportunity to mount a vector control intervention or to prepare for hospital demand. Extensions of this model can also be envisioned for monitoring and predicting other infectious diseases in other geographic areas.

Received October 26, 2007. Accepted for publication August 4, 2008.

Acknowledgments: We thank the reviewers for their relevant comments, and Kristina Talbert-Slagle and Angie Hofmann for editing the revision.

Financial support: P. M. Luz was funded by the Brazilian Government (CAPES), Fulbright, Notsew Orm Sands Foundation, and Garfield Weston. V. M. Mendes received financial support from the Brazilian Government $(\mathrm{CNPq})$ and COPPEAD/UFRJ. C. T. Codeço was partially funded by FIOCRUZ (PDTSP-Dengue). C. J. Struchiner was partially funded by $\mathrm{CNPq}$ and FAPERJ. A. P. Galvani acknowledges funding from the Notsew Orm Sands Foundation and Garfield Weston.

Authors' addresses: Paula M. Luz, Department of Epidemiology and Public Health, Yale University, 60 College Street, New Haven, CT 06511, Tel: 203-777-4671, E-mail: paula.luz@yale.edu. Beatriz V. M. Mendes, Institute of Mathematics/COPPEAD, Rio de Janeiro Federal University, Av. Brigadeiro Trompovisk, Ilha do Fundão, Rio de Janeiro, RJ, Brazil 22221-080, Tel: 55-21-25627910, Fax: 55-2125901095, E-mail: beatriz@im.ufrj.br. Claudia T. Codeço, Program for Scientific Computing, Oswaldo Cruz Foundation, Av. Brasil 4365 , Manguinhos, Rio de Janeiro, RJ, Brazil 21040-360, Tel: 55-21-38361100, E-mail: codeco@fiocruz.br. Claudio J. Struchiner, Program for Scientific Computing, Oswaldo Cruz Foundation, Av. Brasil 4365, Manguinhos, Rio de Janeiro, RJ, Brazil 21040-360, Tel: 55-21-38361109, E-mail: stru@fiocruz.br. Alison P. Galvani, Department of Epidemiology and Public Health, Yale University, 60 College St., New Haven, CT 06511, Tel: 203-785-2642, E-mail: alison.galvani@yale.edu.

\section{REFERENCES}

1. Gubler DJ, 1998. Dengue and dengue hemorrhagic fever. Clin Microbiol Rev 11: 480-496.

2. Halstead SB, 2002. Dengue. Curr Opin Infect Dis 15: 471-476.

3. Guzman MG, Kouri G, 2002. Dengue: an update. Lancet Infect Dis 2: 33-42.

4. Wilson ME, Chen LH, 2002. Dengue in the Americas. Dengue Bulletin 26: 44-61.

5. Torres JR, Castro J, 2007. The health and economic impact of dengue in Latin America. Cad Saude Publica 23 (Suppl 1): S23-S31.

6. Nogueira RM, Araujo JMG, Schatzmayr HG, 2007. Dengue viruses in Brazil, 1986-2006. Rev Panam Salud Publica 22: 358363.

7. Camara FP, Theophilo RL, Dos Santos GT, Pereira SR, Camara DC, de Matos RR, 2007. Regional and dynamics characteristics of dengue in Brazil: a retrospective study. Rev Soc Bras Med Trop 40: 192-196.

8. Nogueira RM, Miagostovich MP, Schatzmayr HG, dos Santos FB, de Araujo ES, de Filippis AM, de Souza RV, Zagne SM, Nicolai C, Baran M, Teixeira Filho G, 1999. Dengue in the state of Rio de Janeiro, Brazil, 1986-1998. Mem Inst Oswaldo Cruz 94: 297-304.

9. Nogueira RM, Schatzmayr HG, de Filippis AM, dos Santos FB, da Cunha RV, Coelho JO, de Souza LJ, Guimaraes FR, de Araujo ES, De Simone TS, Baran M, Teixeira G Jr, Miagostovich MP, 2005. Dengue virus type 3, Brazil, 2002. Emerg Infect Dis 11: 1376-1381.

10. Siqueira JB Jr, Martelli CM, Coelho GE, Simplicio AC, Hatch DL, 2005. Dengue and dengue hemorrhagic fever, Brazil, 1981-2002. Emerg Infect Dis 11: 48-53.

11. Honorio NA, Lourenco-de-Oliveira R, 2001. Frequency of Aedes aegypti and Aedes albopictus larvae and pupae in traps, Brazil. Rev Saude Publica 35: 385-391.

12. Gubler DJ, Kuno G, 1997. Dengue and Dengue Hemorrhagic Fever. New York: $\mathrm{CAB}$ International.

13. WHO, 2004. Using Climate to Predict Infectious Disease Outbreaks: A Review. Geneva: World Health Organization, 55.

14. Arcari P, Tapper N, Pfueller S, 2007. Regional variability in relationships between climate and dengue/DHF in Indonesia. Singap J Trop Geogr 28: 251-272.

15. Barrera R, Delgado N, Jimenez M, Valero S, 2002. Ecoepidemiological factors associated with hyperendemic dengue haemorrhagic fever in Maracay city, Venezuela. Dengue Bull 26: 84-92.

16. Burattini MN, Chen M, Chow A, Coutinho FA, Goh KT, Lopez LF, Ma S, Massad E, 2007. Modelling the control strategies against dengue in Singapore. Epidemiol Infect 136: 1-11.

17. Chadee DD, Shivnauth B, Rawlins SC, Chen AA, 2007. Climate, mosquito indices and the epidemiology of dengue fever in Trinidad (2002-2004). Ann Trop Med Parasitol 101: 69-77.

18. Chowell G, Sanchez F, 2006. Climate-based descriptive models of dengue fever: the 2002 epidemic in Colima, Mexico. J Environ Health 68: 40-44.

19. Corwin AL, Larasati RP, Bangs MJ, Wuryadi S, Arjoso S, Sukri N, Listyaningsih E, Hartati S, Namursa R, Anwar Z, Chandra S, Loho B, Ahmad H, Campbell JR, Porter KR, 2001. Epidemic dengue transmission in southern Sumatra, Indonesia. Trans $R$ Soc Trop Med Hyg 95: 257-265.

20. Depradine C, Lovell E, 2004. Climatological variables and the incidence of dengue fever in Barbados. Int J Environ Health Res 14: 429-441.

21. Focks DA, Barrera R, 2006. Dengue Transmission Dynamics: Assessment and Implications for Control. Report on the Scientific Working Group on Dengue, 2006. Geneva: World Health Organization, 92-109.

22. Keating J, 2001. An investigation into the cyclical incidence of dengue fever. Soc Sci Med 53: 1587-1597.

23. Nakhapakorn K, Tripathi NK, 2005. An information value based analysis of physical and climatic factors affecting dengue fever and dengue haemorrhagic fever incidence. Int J Health Geogr 4: 13.

24. Wu PC, Guo HR, Lung SC, Lin CY, Su HJ, 2007. Weather as an 
effective predictor for occurrence of dengue fever in Taiwan. Acta Trop 103: 50-57.

25. Bangs MJ, Larasati RP, Corwin AL, Wuryadi S, 2006. Climatic factors associated with epidemic dengue in Palembang, Indonesia: implications of short-term meteorological events on virus transmission. Southeast Asian J Trop Med Public Health 37: $1103-1116$.

26. Scott TW, Morrison AC, 2003. Aedes aegypti and the risk of dengue-virus transmission. Takken W, Scott TW, eds. Ecological Aspects for Application of Genetically Modified Mmosquitoes. Dordretch, The Netherlands: FRONTIS, 187-206.

27. Helfenstein U, 1991. The use of transfer function models, intervention analysis and related time series methods in epidemiology. Int J Epidemiol 20: 808-815.

28. Choi K, Thacker SB, 1981. An evaluation of influenza mortality surveillance, 1962-1979. 1. Time-series forecasts of expected pneumonia and influenza deaths. Am J Epidemiol 113: 215226.

29. Farmer RD, Emami J, 1990. Models for forecasting hospital bed requirements in the acute sector. J Epidemiol Community Health 44: 307-312.

30. Milner PC, 1997. Ten-year follow-up of ARIMA forecasts of attendances at accident and emergency departments in the Trent region. Stat Med 16: 2117-2125.

31. Nobre FF, Monteiro AB, Telles PR, Williamson GD, 2001. Dynamic linear model and SARIMA: a comparison of their forecasting performance in epidemiology. Stat Med 20: 3051-3069.

32. Allard R, 1998. Use of time-series analysis in infectious disease surveillance. Bull World Health Organ 76: 327-333.

33. Bowie C, Prothero D, 1981. Finding causes of seasonal diseases using time series analysis. Int J Epidemiol 10: 87-92.

34. Helfenstein U, 1986. Box-Jenkins modelling of some viral infectious diseases. Stat Med 5: 37-47.

35. Tong S, Hu W, 2001. Climate variation and incidence of Ross River virus in Cairns, Australia: a time-series analysis. Environ Health Perspect 109: 1271-1273.

36. Trottier H, Philippe P, Roy R, 2006. Stochastic modeling of empirical time series of childhood infectious diseases data before and after mass vaccination. Emerg Themes Epidemiol 3: 9.

37. Reis BY, Mandl KD, 2003. Time series modeling for syndromic surveillance. BMC Med Inform Decis Mak 3: 2.

38. Earnest A, Chen MI, Ng D, Sin LY, 2005. Using autoregressive integrated moving average (ARIMA) models to predict and monitor the number of beds occupied during a SARS outbreak in a tertiary hospital in Singapore. BMC Health Serv Res 5: 36.

39. Box GEP, Jenkins GM, 1976. Time Series Analysis: Forecasting and Control. San Francisco: Holden-Day.

40. Heiberger RM, Holland B, 2004. Statistical Analysis and Data Display: An Intermediate Course with Examples in S-plus, $R$, and $S A S$. New York: Springer.

41. ADPRJ, 2007. Armazen dos dados da Prefeitura do Rio de Janeiro (City-hall of Rio de Janeiro). Available at: http://www .armazemdedados.rio.rj.gov.br/. Accessed July 20, 2007.

42. SMSRJ, 2007. Secretaria Municipal de Saúde do Rio de Janeiro (Health Department of the city of Rio de Janeiro). Available at: http://www.saude.rio.rj.gov.br/. Accessed June 15, 2007.

43. Heiberger RM, Teles P, 2002. Displays for direct comparison of ARIMA models. Am Stat 56: 131-138.
44. Ljung GM, Box GEP, 1978. Measure of lack of fit in time-series models. Biometrika 65: 297-303.

45. R Development Core Team, 2007. R: A Language and Environment for Statistical Computing. Vienna, Austria: R Foundation for Statistical Computing.

46. Hyndman RJ, 2008. Forecast: Forecasting Functions for Time Series. R package version 1.11. Available at: http://www .robhyndman.info/Rlibrary/forecast/.

47. Hyndman RJ, Khandakar Y, 2008. Automatic time series forecasting: the forecast package for R. J Statis Software.

48. Scott TW, Amerasinghe PH, Morrison AC, Lorenz LH, Clark GG, Strickman D, Kittayapong P, Edman JD, 2000. Longitudinal studies of Aedes aegypti (Diptera: Culicidae) in Thailand and Puerto Rico: blood feeding frequency. J Med Entomol 37: 89-101.

49. Scott TW, Morrison AC, Lorenz LH, Clark GG, Strickman D, Kittayapong P, Zhou H, Edman JD, 2000. Longitudinal studies of Aedes aegypti (Diptera: Culicidae) in Thailand and Puerto Rico: population dynamics. J Med Entomol 37: 77-88.

50. Watts DM, Burke DS, Harrison BA, Whitmire RE, Nisalak A, 1987. Effect of temperature on the vector efficiency of Aedes aegypti for dengue 2 virus. Am J Trop Med Hyg 36: 143-152.

51. Focks DA, Brenner RJ, Hayes J, Daniels E, 2000. Transmission thresholds for dengue in terms of Aedes aegypti pupae per person with discussion of their utility in source reduction efforts. Am J Trop Med Hyg 62: 11-18.

52. da Cunha RV, Dias M, Nogueira RM, Chagas N, Miagostovich MP, Schatzmayr HG, 1995. Secondary dengue infection in schoolchildren in a dengue endemic area in the state of Rio de Janeiro, Brazil. Rev Inst Med Trop Sao Paulo 37: 517-521.

53. Figueiredo LT, Cavalcante SM, Simoes MC, 1990. Dengue serologic survey of schoolchildren in Rio de Janeiro, Brazil, in 1986 and 1987. Bull Pan Am Health Organ 24: 217-225.

54. Honorio NA, Silva Wda C, Leite PJ, Goncalves JM, Lounibos LP, Lourenco-de-Oliveira R, 2003. Dispersal of Aedes aegypti and Aedes albopictus (Diptera: Culicidae) in an urban endemic dengue area in the State of Rio de Janeiro, Brazil. Mem Inst Oswaldo Cruz 98: 191-198.

55. de Lima-Camara TN, Honorio NA, Lourenco-de-Oliveira R, 2006. Frequency and spatial distribution of Aedes aegypti and Aedes albopictus (Diptera: Culicidae) in Rio de Janeiro, Brazil. Cad Saude Publica 22: 2079-2084.

56. Maciel-De-Freitas R, Codeco CT, Lourenco-De-Oliveira R, 2007. Daily survival rates and dispersal of Aedes aegypti females in Rio de Janeiro, Brazil. Am J Trop Med Hyg 76: 659665.

57. Maciel-de-Freitas R, Marques WA, Peres RC, Cunha SP, de Oliveira RL, 2007. Variation in Aedes aegypti (Diptera: Culicidae) container productivity in a slum and a suburban district of Rio de Janeiro during dry and wet seasons. Mem Inst Oswaldo Cruz 102: 489-496.

58. Gubler DJ, 2002. Epidemic dengue/dengue hemorrhagic fever as a public health, social and economic problem in the 21 st century. Trends Microbiol 10: 100-103.

59. Teixeira MG, Costa MCN, Guerra Z, Barreto ML, 2002. Dengue in Brazil: situation-2001 and trends. Dengue Bull 26: 70-76.

60. de Souza IC, Vianna RP, de Moraes RM, 2007. Modeling of dengue incidence in Paraiba State, Brazil, using distributed lag models. Cad Saude Publica 23: 2623-2630. 